Itinéraires Itinéraires

Littérature, textes, cultures

2011-2| 2011

Le concept de mémoire

\title{
Parémie et mémoire
}

\section{Guy Achard-Bayle et Bertille Schneider}

\section{(2) OpenEdition}

Journals

Édition électronique

URL : https://journals.openedition.org/itineraires/118

DOI : 10.4000/itineraires. 118

ISSN : 2427-920X

Éditeur

Pléiade

\section{Édition imprimée}

Date de publication : 1 juillet 2011

Pagination : 55-77

ISBN : 978-2-296-54673-8

ISSN : 2100-1340

Référence électronique

Guy Achard-Bayle et Bertille Schneider, « Parémie et mémoire », Itinéraires [En ligne], 2011-2 | 2011, mis en ligne le 13 janvier 2014, consulté le 08 juin 2021. URL : http://journals.openedition.org/ itineraires/118; DOI : https://doi.org/10.4000/itineraires.118

\section{$\Theta \oplus \Theta \Theta$}

Itinéraires est mis à disposition selon les termes de la licence Creative Commons Attribution - Pas d'Utilisation Commerciale - Pas de Modification 4.0 International. 


\title{
Parémie et mémoire
}

\begin{abstract}
This article explores the collective (cultural and linguistic) dimension of memory as realized in proverbs. These simple forms are called "frozen" because they are supposed to be the preservers of common sense in terms of intersubjectivity and mutual understanding. We try to show, however, by means of a questionnaire, how the syntactic and semantic structure of proverbial expressions can have a dynamic dimension manifested in a paremic competence of interlocutors who are able to understand, interpret, adapt or create "proverbial forms" that may not actually exist.
\end{abstract}

Keywords : paremiology, proverbs, intersubjectivity, intercomprehension, idiolect Mots clés : parémiologie, proverbes, intersubjectivité, intercompréhension, idiolecte

\section{Introduction}

Cette contribution fait suite à une autre étude commune (2010) : celle-ci visait l'analyse syntaxique et sémantique des relations intégratives et implicatives de proverbes binaires (i.e. en deux propositions $P+Q$ ), avec ou sans « marqueur d'intégration » (ou « cheville de liage » suivant Le Goffic 2002) : Qui P, $Q$, Si P, Q, Quand P, $Q$ (pour l'hypotaxe ou la subordination) et $P, Q$ (pour la parataxe).

Nous disions à plusieurs reprises dans cette étude que nous remettrions à plus tard l'étude sémantico-cognitive de la relation implicative entre les deux prédicats $\mathrm{P}$ et $\mathrm{Q}$. Mais nous annoncions plusieurs pistes de travail, que nous allons commencer par rappeler.

La binarité très fréquente (sinon exclusive) des énoncés parémiques [EP] résulte de divers procédés de parallélisme, ou de mise en correspondance, ou d'écho, qui sont à la fois formels (syntaxiques, prosodiques) et pragma-sémantiques (lexicaux, rhétoriques). Ils ont pour effet (i) « de lier encore plus fortement les constituants de l'énoncé » et (ii) « de déclencher le processus interprétatif parémique, notamment en l'absence d'organisation 
hypotaxique "), comme avec ces deux premiers exemples (ex. 22 et 24 d'Achard-Bayle et Schneider 2010) :

(1) Jeune saint, vieux démon.

(2) Suis le plaisir, il te fuira, fuis le plaisir, il te suivra.

On voit ainsi se profiler notre problématique présente : il y a dans la structure (signifiante comme signifiée) des EP une fonction cognitive mémorielle évidente, donc essentielle; précisément, elle est à la fois mnémotechnique et poïétique (ou relatives à la mnemosis et à la mimesis). Autrement dit, considérant qu'il existe une compétence parémique chez les locuteurs d'une langue-culture, en compréhension-réception, celle-ci s'accompagne, en production-expression, d'une performance parémique, qui permet à l'un desdits locuteurs de créer des énoncés pseudo-parémiques, et à ses interlocuteurs de les interpréter; c'est ce que Gouvard (1996 : 48) appelle des « formes proverbiales » :

Les formes proverbiales sont des énoncés tels que « À pluie battante, eaux combattantes » ou « Celui qui bêche tôt cueillera », dont tout locuteur reconnaît intuitivement qu'ils ressemblent à des proverbes ou qu'ils pourraient être des proverbes qu'ils ne connaissent pas, tout en étant des créations individuelles (ces deux citations sont de mon cru).

Dans notre première étude à deux, donc, nous en restions à ce constat et à cette annonce :

Notre approche était essentiellement descriptive, même si la diversité sémantique soulignée nous a conduits à de nombreux tests d'interprétation. Il nous reste toutefois, pour mieux comprendre les conditions, les circonstances et les effets de l'interprétation (ou de la compétence) parémique, à poursuivre dans une perspective davantage cognitive. (Achard-Bayle et Schneider 2010, conclusion)

C'est ce que nous allons donc faire ici; et nous le ferons en deux parties.

Dans une première partie nous nous attacherons à cadrer cognitivement notre objet d'étude et la problématique «bi-mémorielle » que nous avons choisie pour son traitement, mnémonique et mimétique. Nous repartirons des notions d'écho et de généricité que l'on trouve, l'une notamment chez Gouvard (1996), l'autre notamment chez Kleiber (1999, 2000), mais plus précisément encore et avant lui chez les linguistes cognitivistes américains qui traitent le sujet en termes d' " esprit littéraire » (Turner 1996) et de « lieux communs » (de « modèles idéalisés », d' « espaces génériques »: Lakoff 1987, Fauconnier 1984).

Dans la seconde partie, nous partirons de l'hypothèse développée précédemment (2010) d'un « moule syntaxique binaire » pour présenter les résultats d'une enquête de terrain; il s'agit pourrait-on dire d'une enquête d' " opinion », autrement dit et jusqu'à un certain point de « doxa », destinée 
à vérifier non seulement l'hypothèse en question, mais plus précisément la compétence parémique d'un public bien défini et particulier, sur fond de mémoire collective culturelle, certes, mais plus ou moins partagée.

\section{Des Vies minuscules. Énoncés parémiques et cognition}

Pour introduire à cette étude d'opinion au sens de la doxa ou de la croyance, nous dirons pour présenter la problématique générale de notre contribution que les proverbes sont des « vies minuscules ». Nous allons pour commencer nous attarder quelque peu sur ce titre qui peut paraître inattendu; nous l'expliquerons de deux manières. La première nous permettra de situer notre problématique dans un cadre épistémologique large : celui de la linguistique cognitive; la seconde dans un cadre sinon plus limité du moins plus étroit et davantage centré sur la problématique des actes : celui de la mémoire. La cognition, plus ou moins sociale, collective et culturelle, nous permettra de faire le lien entre ces deux approches des proverbes.

\subsection{Les proverbes dans un cadre cognitif large}

Les proverbes sont des formes courtes, même si leur longueur est variable, et si la tendance a pu être, diachroniquement, à leur réduction (Achard-Bayle et Schneider 2010). Ce sont des formes courtes au sens strictement morphologique ou morphosyntaxique, mais aussi au sens des formes simples de Jolles (1972), autrement dit des genres ou types de textes courts.

Cette approche générique textuelle de la forme non seulement convient bien nous semble-t-il à l'environnement, littéraire et linguistique, où notre étude prend place, mais encore elle permet d'ouvrir la réflexion à la problématique cognitive, c'est-à-dire de la situer, comme on l'a dit plus haut, dans un cadre épistémologique large; que nous allons présenter, en deux sections de nouveau.

\subsubsection{L'Esprit littéraire - The Literary Mind}

L'ouvrage qui nous servira ici de référence est celui de Turner (1996), pour qui « notre esprit est littéraire »; mais l'avis est partagé, de Lakoff et Johnson (1981) à Gibbs (2008). Cela veut dire assez simplement que notre cognition humaine, façonnée par des millénaires de vie sociale, et de codes et de rites culturels partagés, donc de vieille mémoire collective perdue dans les origines de l'espèce (celle de l'homo sapiens sapiens ou sapiens narrans suivant Victorri 2006a), fonctionne suivant des images, des schémas, des modèles, d'organisation et de représentation, et, ceci est très important pour la linguistique cognitive californienne, de création sémantique, que l'on qualifie de littéraire, alors même que, comme les métaphores de Lakoff 
et Johnson, ces images, schémas ou modèles envahissent, mentalement parlant, notre vie quotidienne.

Les métaphores, donc, en sont une démonstration; mais on peut aller plus loin, avec Turner (1996), pour voir dans l'organisation argumentale d'un certain nombre de propositions, ou encore dans les liens qui unissent macrosyntaxiquement, autrement dit en termes de lexique-grammaire et de syntaxe fonctionnelle, les arguments qui composent ces propositions, une image, l'icone de représentations narratives (voire romanesques ou dramatiques, suivant notamment le temps choisi) :

(3) La Bourse s'est effondrée [s'effondre].

(4) La maison est tombée [tombe] en ruines.

Il s'agit là, pour Turner (47-48), de « stories without animate actors » ou encore de « spatial stories ». Ainsi, dans l'exemple (4), une histoire spatiale de « chute » (falling) est projetée sur une autre histoire spatiale, assez différente, de tuiles qui se cassent, de peintures qui s'écaillent, de vitres qui se fendent.

Nous donnerons également l'exemple, avec Turner, du (goût du) mélange, du mixage (blending : entre projections d'espace à espace et réduction sémantique des liens qui les unissent) qui (re-)configure des identités évolutives, des êtres hybrides (la licorne), ou encore des mondes hypothétiques et contrefactuels (« irréel du passé »).

Sur cette base, on peut dire que les proverbes sont de « very short stories ». Mais si celles-ci peuvent être « sans acteurs », ce n'est que superficiellement, par effacement ou remplacement figuré, autrement dit par projection métaphorique. D'où notre deuxième section.

\subsubsection{Les proverbes sont des histoires en raccourci}

Les proverbes sont des formes courtes et génériques, certes, mais pas seulement ou toujours des récits. Inversement, La Bourse s'est effondrée n'est pas un EP : à cause de la spécificité du SN et du temps verbal. Il faudrait ce que Kleiber (2006) appelle une « montée en généricité », et un tour particulier (nous y revenons), pour déclencher l'interprétation parémique.

Pour autant toute proposition ou phrase au présent et au défini ou à l'indéfini génériques n'est pas un EP : voir les maximes, les énoncés de vérité générale. Il faut donc une montée en généricité et si l'on peut dire une « échappée » en métaphoricité, ou une « entrée » dans l'esprit littéraire. Pour cela il faut se référer à un principe de mise en correspondance, de blending et de projection : la « grande chaîne des êtres » (ou de l'étant) que Kleiber ne cite pas, qui vient de Lakoff et Turner (1989), et qu'eux-mêmes empruntent à Lovejoy (1936) : The Great Chain of Being. 


\subsection{Les proverbes dans un cadre cognitif restreint}

Nous commençons par éclaircir ce mystère des « vies minuscules » :

\footnotetext{
À Mourioux dans mes premiers âges, il arrivait lorsque j'étais malade ou seulement inquiet, que ma grand-mère pour me divertir allât chercher les Trésors. J'appelais ainsi ces deux boîtes de fer-blanc naïvement peintes et cabossées qui avaient jadis contenu des biscuits, mais qui recelaient alors de tout autres nourritures : ce qu'en tirait alors ma grand-mère, c'était des objets dits précieux et leur histoire, de ces bijoux transmis qui sont mémoires aux petites gens... (Pierre Michon, Vies minuscules, Paris, Gallimard, 1984, rééd. coll. « Folio », 2009, p. 33)
}

Il s'agit d'un extrait de l'ouvrage éponyme de Michon. Nous nous intéresserons donc, une nouvelle fois, à ce que nous avons pu appeler des « réalités conceptuelles » (Achard-Bayle 2008) : elles sont fondamentalement issues d'un mélange/blending matière-esprit, telles ces entités spatiales à la " géographie invisible », suivant Giono (Jean le Bleu, 1932, rééd. Le Livre de Poche, 1974, p. 26); c'est-à-dire telles qu'on se les figure. Mais ici, nous nous intéresserons plus qu'à l'idée figurée, à l'image décalée ou différée dans l'espace : c'est ce que représentent les lieux de mémoire comme « lieux communs », autrement dit les « lieux de commune mémoire ».

Ceux-ci, s'ils ne sont pas tous de commémoration, sont des « entreprises de remémoration collective », parfois suivant des "procédés mimétiques » (comme les récits des origines ou les premiers récits selon Victorri 2006a: 123).

Pour ce qui est des EP, il s'agit sans doute d'une survivance d'une forme d'expression qu' on peut dire archaïque ou archaïsante qui marque cependant bien le passage à ou un premier stade de la « culture mythique » (ou méta-mimétique, si l'on adapte les deux phases et réinterprète le passage de la culture mimétique à la mythique de Donald 1991, cité par Victorri 2006a : 128).

On peut aussi voir dans les EP des lieux de « external symbolic storage », « external » en ce qu'ils sont, comme l'écriture à laquelle Donald (1991) se réfère, fixés, figés, de par leur fonction mémorielle. En même temps, ils fixent, figent, dans un moule formel et culturel, cette " relativité des expériences sans fin » de la vie profane, dont a pu parler Eliade (1957/1965), afin d'en faire en quelque sorte une réalité objectivée sinon sanctifiée.

Eliade montre ainsi que les sociétés humaines construisent des lieux physiques qui non seulement matérialisent l'espace du sacré, en en faisant un espace sacré, mais le plus souvent montent (vers Dieu ou les dieux). On obtient ainsi et une schématisation, en fait une verticalisation de l'espace, et une valorisation de cet espace dans ses hautes sphères (ce qui n'est pas sans écho avec l'idée de notre montée en généricité). Et ce sont des représentations que l'on retrouve de la scala naturae (Aristote) à la grande 
chaîne des êtres (jusqu'au XVII ${ }^{\mathrm{e}}$ siècle chez Leibniz, Spinoza, Descartes : cf. Suber 1997); que l'on retrouve même au XVIII ${ }^{\mathrm{e}}$ siècle quand la philosophie s'est en quelque sorte sécularisée (cf. Bonnet et annexes).

Ayant ainsi fait la transition entre les deux sens que nous voulions donner à « vies minuscules », nous revenons maintenant à notre démonstration sur la montée en généricité, laissée en suspens à la fin de la section 1.1. :

Il faut donc une montée en généricité, et une montée en métaphoricité, ou une entrée dans l'esprit littéraire. Et pour cela il faut se référer à un principe de mise en correspondance, de blending et de projection : la grande chaîne des êtres...

Pour Lakoff et Turner (1989), cette grande chaîne des êtres [GCE] est l'arrière-plan ontologico-culturel qui permet de réaliser des projections et donc des assimilations ou des appariements (métaphoriques) entre différents maillons de la chaîne, donc entre entités du monde, même si tout rapprochement, toute assimilation n'est pas possible : à la question que Lakoff et Turner (se) posent « Can anything be anything? », leur réponse est carrément « non »!

Cette GCE, nous l'avons dit, fait inévitablement écho à la montée en généricité, d'une part, d'autre part, à la manière dont les linguistes cognitivistes californiens schématisent eux-mêmes les espaces mentaux et leur interrelation et leur interaction (suivant les principes ou les opérations de bridging et mapping). L'espace générique (generic space), celui qui gouverne les espaces d'entrée (input) et l'espace final de mélange et de création de sens nouveau (new meaning emerging space), les chapeaute et les domine tous. Il est bien ainsi, iconiquement, celui qui donne les instructions venues d'en haut, de schémas, de modèles cognitifs idéalisés, qui permettent de réaliser et de justifier inférentiellement et contextuellement les créations nouvelles, de les insérer dans des cadres ou des moules formellement et encyclopédiquement reconnus ou reconnaissables : ce qui est une autre forme de mémoire et de mimesis.

C'est ce qui se passe d'une certaine manière avec la création des « formes proverbiales », qui doivent pouvoir se rattacher à une forme certes mais aussi à une situation générique (prototypique) attestée ou attestable, et qui combinent ainsi l'inédit à l'air de famille.

\section{La compétence parémique : entre mémoire collective et culturelle}

\subsection{Le proverbe : une catégorie définie?}

Cette idée de moule proverbial n'est pourtant pas développée en littérature scientifique, si l'on s'intéresse aux tentatives de définition du proverbe en termes linguistiques. Cette dernière est établie par un listage de traits parémiques, que plusieurs spécialistes, notamment J.-C. Anscombre, ont 
critiqué, remanié, pour catégoriser ce type d'énoncés. Selon lui (2000 : 7), il existerait une « vulgate » sur le proverbe, que l'auteur résume comme suit :

Le proverbe est :

(i) une entité phrastique autonome ;

(ii) doué d'un contenu sentencieux (la valeur « prescriptive» du proverbe) ;

(iii) l'expression d'une vérité générale, fondée sur l'expérience ;

(iv) bref, populaire (« vulgaire », selon certains auteurs) et généralement métaphorique ;

(v) bi-membre, souvent pourvu de rimes et d'éléments répétitifs, lesquels sont chargés de faciliter sa mémorisation;

(vi) ancien, et se transmet fidèlement de génération en génération. Il s'agit d'un genre essentiellement oral, et de cette fidèle transmission découle son statut de tournure figée, ainsi que l'abondante présence de structures archaïsantes. Le proverbe relève exclusivement d'un lexique spécial (on doit apprendre les proverbes par cœur).

Dans le cadre qui nous intéresse ici, on retiendra en particulier deux traits définitoires, d'une part la « sagesse populaire » (synthétisant le côté populaire, anonyme et ancien), d'autre part la « fixité formelle » (ou pérennité formelle) de l'énoncé proverbial. Si pour nombre de spécialistes ces deux traits sont indissociables de la notion de proverbe, pour d'autres en revanche, ils sont réfutables, ou à tout le moins discutables. Ainsi, le caractère populaire et anonyme de l'origine du proverbe est contesté : certains énoncés proverbiaux émanent d'auteurs connus (Ainsi en est-il de Rien ne sert de courir, il faut partir à point dont J.-C. Anscombre (2000 : 11-12) rappelle qu'il est tiré d'une fable de La Fontaine ${ }^{1}$.) L'ancienneté est contestée par C. Schapira (2000:83) qui écrit sans ambiguïté : « D'autres proverbes $[\ldots]$ sont en train de se créer de nos jours sous nos yeux. "; ces proverbes ou à tout le moins ces énoncés proverbiaux, venant, d'après elle, de la publicité, de mots d'hommes ou de femmes politiques (On ne tire pas sur une ambulance venant de F. Giroud), du domaine du sport (On ne change pas une équipe qui gagne), par exemple ${ }^{2}$.

La «fixité 》 ou la «pérennité 》 formelle est contredite par J.-C. Anscombre (2003) et l'est aussi par C. Schapira (2000 : 85-86).

La plupart des proverbes actuels ont initialement connu plus d'une version [...] jusqu'à ce qu'ils se stabilisent sous la forme standard en usage de nos jours. Aujourd'hui encore il est possible de trouver des proverbes à forme hésitante : Deux sûretés / Deux précautions valent mieux qu'une; Ne réveillez pas le chien / le chat qui dort.

1. Un auteur connu, même si le passage du vers de la fable au statut de proverbe « modifie le statut énonciatif de l'énoncé considéré ", dans la mesure où ce n'est plus La Fontaine qui est l'énonciateur. Il n'empêche que l'origine n'est plus populaire, n'est plus anonyme. Cf. aussi C. Schapira (2000:83).

2. Les exemples se trouvent dans C. Schapira (2000:87). 


\subsection{Le proverbe en usage}

Il faut donc chercher ailleurs que dans ces tentatives de définition du proverbe pour expliquer comment et pourquoi l'énoncé parémique, profondément ancré dans la mémoire, est reconnu comme tel par tout locuteur et loin de ne présenter qu'une seule forme attestée par les dictionnaires, son emploi en discours est sujet à variantes, qui n'altèrent en rien le sens profond qu'il véhicule. Selon nous, c'est l'idée de l'existence d'un moule proverbial (structure profonde) sur lequel tout locuteur pourrait se calquer pour fabriquer ou interpréter des proverbes, qui permet d'expliquer la diversité linguistique d'un énoncé dont le sens profond est connu de tous. Ce sens profond est commun à tous (mémoire collective), quelle que soit la culture d'origine ou d'adoption du locuteur. C'est la manifestation de ce sens profond en discours qui variera en fonction de la culture voire de l'idiolecte des uns et des autres.

Par exemple, on peut facilement comprendre l'intention du locuteur qui nous dira : Celui qui s'est brûlé avec la tchorba ${ }^{3}$ souffle jusque dans le yaourt (traduction d'un proverbe roumain) bien que la culture ici référencée ne soit pas notre culture d'origine; on pourra spontanément rapprocher l'énoncé du proverbe Chat échaudé craint l'eau froide propre à notre idiolecte. C'est, selon nous, le sens profond ancré dans la mémoire collective d'un peuple, d'une culture et qui se manifesterait en laissant une part de liberté au sujet parlant dans la formulation choisie (expériences culturelle, collective et individuelle sur lesquelles nous reviendrons plus tard).

L'existence de cette compétence parémique a été démontrée via les résultats d'une enquête auprès d'informants non spécialistes (67 retours). Le panel de population interrogée - de sexe, d'âge, catégorie socioprofessionnelle différents mais ayant en commun le rattachement à une région donnée : la Lorraine (soit par le lieu de naissance, de résidence actuel ou parce qu'ils y ont résidé pendant une période supérieure à une année) nous permet, par ses réponses, non seulement de montrer les limites d'une approche immuable de la définition du « concept proverbe », mais aussi et surtout de prouver l'existence de cette compétence parémique, qui est étroitement liée à la mémoire culturelle collective et individuelle de tout locuteur intervenant dans le choix des proverbes en usage.

Nous présentons ci-après cette enquête.

\section{a. L'enquête : les questions}

(I) Citez les dix proverbes qui vous semblent les plus représentatifs de ce type d'énoncés.

(II) Proposez cinq énoncés de votre composition, qui pourraient faire office de proverbes.

3. La tchorba est une soupe de boulettes de viande très relevée (recette bulgare). 
(III) Complétez chacun de ces énoncés selon la variante que vous connaissez ou employez.

1. À la Sainte-Catherine,

2. Il faut battre le fer,

3. N'éveillez pas. qui dort.

4. À la Sainte-Luce,

5. S'il pleut à la Saint-Médard

À moins que Saint-Barnabé.....

(IV) Dans le tableau ci-dessous, indiquez d'une croix à quelle catégorie d'énoncés les exemples (1) à (15) appartiennent parmi : Proverbe - Dicton - Maxime - Autre. Si pour certains énoncés votre choix s'oriente vers la réponse " autre », veuillez alors préciser à quel type de phrase les exemples en question correspondent.

1. A pisser contre le vent, on mouille sa chemise.

2. Rien ne sert de courir, il faut partir à point.

3. Dans la poussière on voit les poules, avant l'orage qui se roulent.

4. Le cour a ses raisons que la raison ne connaît point.

5. Frauder, c'est risquer.

6. Le zèbre ne se défait pas de ses rayures.

7. Il y a loin de la coupe aux lèvres.

8. Quand le chat se débarbouille, bientôt le temps se brouille.

9. Qui est roux, aime les choux.

10. En avril ne te découvre pas d'un fil, en mai fais ce qu'il te plaît.

11. Un pour tous, tous pour un.

12. Nul n'est prophète en son pays.

13. Quand il tonne en mai, les vaches ont du lait.

14. Beaucoup de bruit, peu de fruit.

15. Qui veut retenir, laisse tout échapper.

(V) Si vous deviez proposer une définition du mot « proverbe » sans pouvoir citer d'exemple(s), quels seraient, selon vous, les cinq « mots clés » les plus caractéristiques de la notion.

\section{b. L'enquête : quelques résultats probants}

Nous nous limiterons ici à présenter quelques résultats des questions I, II et III afin de montrer l'existence d'une compétence parémique et donc l'inscription du sens proverbial profond dans un savoir partagé par tous. Ceux-ci prouvent par là même la différence réelle entre les caractéristiques répertoriées par la vulgate et du même fait enseignées à tout locuteur comme étant la définition du proverbe prototypique et la représentation réelle que tout locuteur a de la notion.

(I) Parmi les 390 énoncés relevés dans les réponses, nous proposons ici une partie des occurrences les plus fréquentes. 


\begin{tabular}{|c|c|c|c|}
\hline \multicolumn{3}{|r|}{ Énoncé proverbial } & Occurrences \\
\hline \multirow{3}{*}{5} & forme attestée & Qui vole un œuf vole un bœuf. & \multirow{3}{*}{16} \\
\hline & \multirow{2}{*}{ variantes } & Qui vole un œuf, vole un bœuf. & \\
\hline & & Qui vole un bœuf vole un œuf. & \\
\hline \multirow{3}{*}{6} & forme attestée & L'habit ne fait pas le moine. & \multirow{3}{*}{16} \\
\hline & \multirow[b]{2}{*}{ variantes } & L'habit fait pas le moine. & \\
\hline & & $\begin{array}{l}\text { L'habit ne fait pas le moine mais les belles plumes font le } \\
\text { bel oiseau. }\end{array}$ & \\
\hline \multirow{4}{*}{7} & forme attestée & Tant va la cruche à l'eau, qu'à la fin elle se casse. & \multirow{4}{*}{13} \\
\hline & \multirow{3}{*}{ variantes } & Tant va la cruche à l'eau, qu'à la fin elle se casse. & \\
\hline & & Tant va la cruche à l'eau qu'elle se casse. & \\
\hline & & Tant va la cruche à l'eau qu'elle se brise. & \\
\hline \multirow{4}{*}{8} & forme attestée & Rien ne sert de courir, il faut partir à point. & \multirow{4}{*}{13} \\
\hline & \multirow{3}{*}{ variantes } & Rien ne sert de courir il faut partir à point. & \\
\hline & & Rien ne sert de courir, il faut partir à temps. & \\
\hline & & Rien ne sert de courir, mieux vaut partir à point. & \\
\hline \multirow{3}{*}{9} & forme attestée & Pierre qui roule n'amasse pas mousse. & \multirow{3}{*}{12} \\
\hline & \multirow{2}{*}{ variantes } & Pierre qui roule, n'amasse pas mousse. & \\
\hline & & Pierre qui roule n'amasse pas de mousse. & \\
\hline \multirow{2}{*}{10} & forme attestée & Tout vient à point à qui sait attendre. & \multirow{2}{*}{10} \\
\hline & variante & Tout arrive à point pour qui sait attendre. & \\
\hline 11 & forme attestée & Qui sème le vent récolte la tempête. & 9 \\
\hline 12 & forme attestée & Noël au balcon, Pâques aux tisons. & 9 \\
\hline 13 & forme attestée & La nuit tous les chats sont gris. & \multirow{2}{*}{9} \\
\hline 15 & variante & La nuit tous, les chats sont gris. & \\
\hline
\end{tabular}

Ce tableau récapitulatif montre bien que la fixité formelle de l'énoncé n'est pas un trait fiable. Les occurrences recensées présentent, dans la construction de la forme écrite, des variantes de la forme attestée, au niveau :

- de la ponctuation : présence ou non d'une virgule, ce qui se manifeste oralement par la réalisation ou non d'une pause - (5), (6), (8), (9), (13); - de la construction syntagmatique :

- présence ou non de préposition : énoncés (9), (10),

- négation : terme négatif élidé ou non - (6),

- du choix du lexique : emploi de synonymes - (7), (8).

Les énoncés proposés sont cependant sémantiquement équivalents (exception faite de (5) - variante 2); ils se rapprochent tous de l'énoncé attesté, mais varient selon la mémoire culturelle et l'idiolecte de chacun.

(II) La compétence parémique en création s'analyse dans le test de la capacité de composition d'énoncés proverbiaux. Parmi tous les énoncés qui ont été recensés, certains sont inventés de toute pièce :

(14) Si les fruits ne sont pas mûrs, ne les cueille pas.

(15) Pingouins dans les champs, hiver méchant.

(16) Pas de bras, pas de chocolat. 
(17) Si le lapin dort deux fois, relève-toi à petits pas.

(18) Qui prend le train souvent, devient gréviste consentant.

D'autres en revanche sont clairement calqués sur des proverbes attestés; leurs auteurs en créent de nouveaux soit par détournement, soit par compilation des deux proverbes existant :

(19) Si la goutte d'eau met le feu aux poudres, l'étincelle fait déborder le vase.

(20) Un homme averti vaut deux pierres qui roulent.

(21) Qui dort, dort.

Dans tous ces exemples, l'existence d'un moule proverbial est probante. Les «nouveaux proverbes » sont clairement à rapprocher de proverbes existants dans notre patrimoine culturel. Si le sens littéral de ces derniers n'est pas forcément identifiable ou à tout le moins pertinent pour chacun des énoncés, en revanche, les caractéristiques formelles (construction syntaxique binaire), poétiques (rythme prosodique, jeu des sonorités (rimes, allitérations, assonances)) et sémantique (relation implicative si $P$, alors $Q$ ayant valeur de mise en garde, conseil) sont toujours respectées.

(III) Si l'on prend l'exemple d'un ou deux énoncés proverbiaux soumis aux informants, et qu'on leur demandait de compléter, là encore, les résultats sont significatifs : d'une part ils vont dans le sens de la non-fixité formelle du proverbe, d'autre part ils nous proposent un aperçu des variantes régionales et surtout permettent d'analyser en quoi cette part de mémoire collective ancrée profondément se distingue ou non de la mémoire « culturelle » qui elle, laisse une part de liberté aux sujets parlants :

\begin{tabular}{|l|c|}
\hline \multicolumn{1}{|c|}{ Forme proverbiale attestée } & Nb de variantes \\
\hline Il faut battre le fer tant qu'il est chaud. & 7 \\
\hline À la Sainte-Catherine, tout bois prend racine. & 14 \\
\hline N'éveillez pas le chat qui dort. & 11 \\
\hline À la sainte Luce, le jour croît du saut d'une puce. & 27 \\
\hline $\begin{array}{l}\text { S'il pleut à la St Médard, il pleut 40 jours plus tard, à moins que St Barnabé } \\
\text { ne lui casse le nez. }\end{array}$ & 30 \\
\hline
\end{tabular}

Le nombre de variantes proposées par les informants pour seulement cinq énoncés parémiques est très important et prouve bien la fixité formelle du proverbe.

Voici quelques variantes répertoriées :

\begin{tabular}{|c|l|c|}
\hline $\mathbf{2 2}$ & \multicolumn{1}{|c|}{ À la Sainte-Catherine... } & Fréquence \\
\hline $\mathrm{a}$ & tout bois prend racine. & 20 \\
\hline $\mathrm{b}$ & mets ta capeline. & 1 \\
\hline $\mathrm{c}$ & l'arbre prend racine. & 1 \\
\hline $\mathrm{d}$ & tout le monde fait mine. & 1 \\
\hline $\mathrm{e}$ & tout prend racine. & 13 \\
\hline
\end{tabular}




\begin{tabular}{|c|l|c|}
\hline $\mathbf{2 2}$ & \multicolumn{1}{|c|}{ À la Sainte-Catherine... } & Fréquence \\
\hline f & les arbres prennent racine. & 2 \\
\hline g & tout arbre prend racine. & 7 \\
\hline h & tout replant prend racine. & 1 \\
\hline i & la végétation prend racine. & 1 \\
\hline j & plante n'importe quoi, tout fait racine. & 1 \\
\hline k & les jeunes filles se coiffent. & 1 \\
\hline l & les femmes sont des coquines. & 1 \\
\hline m & pour tout l'hiver fait ta farine. & 1 \\
\hline n & tout arbre reprend racine. & 1 \\
\hline
\end{tabular}

\begin{tabular}{|c|l|c|}
\hline $\mathbf{2 3}$ & \multicolumn{1}{|c|}{ Il faut battre le fer... } & Fréquence \\
\hline a & tant qu'il est chaud. & 42 \\
\hline b & pendant qu'il est chaud. & 10 \\
\hline c & avant qu'il soit chaud. & 1 \\
\hline d & quand il est chaud. & 7 \\
\hline e & pendant qu'il est encore chaud. & 1 \\
\hline f & tant qu'il est encore chaud. & 4 \\
\hline g & avant qu'il refroidisse. & 1 \\
\hline
\end{tabular}

Si le sens profond du proverbe est dans la plupart des cas bien conservé, ou plutôt la forme proverbiale originale ancrée dans la mémoire sémantique (mémoire collective) des informants, les variantes en usage laissent une certaine liberté au locuteur, qu'elle soit syntaxique (propositions relatives (23d) ou subordonnées), lexicale (choix du lexique (23g)) ou culturelle ((22k) référence à la tradition de la Sainte-Catherine $\left.{ }^{4}\right)$.

Pour autant, la portée de l'énoncé n'est en général pas ou peu altérée (exception faite d'énoncés tels que (21)).

L'enquête nous a permis de recenser d'une part les variations qui existent entre la forme standard attestée par les dictionnaires parémiques et les variantes des formes en usage, et d'autre part parmi les formes en usage, les variantes à l'intérieur d'un panel de la population d'une même région. Ce recensement ne fait qu'étayer notre thèse de l'existence d'une compétence parémique.

\subsection{Compétence parémique et mémoire}

Cette compétence parémique se manifeste donc en création comme en réception. C'est selon nous la structure profonde ancrée dans la mémoire

4. La fête de la Sainte-Catherine est une tradition qui remonte au Moyen Âge. Le 25 novembre, les filles de 25 ans qui n'étaient pas encore mariées avaient le privilège de mettre des coiffes ou des chapeaux sur les statues de sainte Catherine, patronne des jeunes filles à marier, et priaient la sainte de leur donner un bon mari. Actuellement, la catherinette ne va plus dans les églises mais arbore un extravagant chapeau jaune et vert et se trouve à l'honneur toute la journée du 25 novembre. 
collective et qui se manifesterait en langue en laissant une part de liberté au sujet parlant, qui anime cette compétence.

Si l'on applique ce postulat à l'analyse exposée précédemment, la compétence parémique de chaque individu aurait comme point de départ un « stock de proverbes » appartenant à son idiolecte, acquis par apprentissage au même titre que d'autres formes linguistiques telles que les expressions figées (transmises par héritage culturel), et qui évolue avec l'expérience. Ce «stock de proverbes » présente de nombreuses similitudes en fonction de la région d'origine et ou d'appartenance et son développement est donc étroitement lié à la mémoire culturelle et collective.

Quand on pense ou parle proverbe, on va forcément convoquer des idées (espaces mentaux), des concepts ancrés dans la culture - connaissances encyclopédiques. Nous faisons ici référence au développement sur le contenu métaphorique proposé plus haut ainsi qu'aux études de J.-F. Rastier et Fauconnier (reprises par P. Gréa) sur le sujet.

Mémoire collective et mémoire culturelle ne convoquent pas les mêmes savoirs. La mémoire collective ou sémantique est ancrée internationalement, c'est-à-dire qu'elle permet à tout locuteur de convoquer les mêmes savoirs encyclopédiques, ou à tout le moins d'activer les mêmes concepts. Elle est étroitement liée à la compétence parémique en réception; voyons ce rapport étroit si l'on reprend le proverbe cité plus haut :

(24) Celui qui s'est brûlé avec la tchorba souffle jusque dans le yaourt $\downarrow$

Le locuteur reconnaît l'énoncé comme étant une forme proverbiale

La provenance du proverbe n'est pas inhérente à sa culture d'origine $\downarrow$

Il convoque dans son savoir encyclopédique les concepts « brûler » et « yaourt »

$$
\text { Brûler }=\text { chaud } / \text { yaourt }=\text { froid }
$$

L'idée de méfiance : quand on se brûle, on se méfie même d'un plat froid

Une fois cette opération sémantique opérée, le locuteur peut chercher dans sa mémoire culturelle l'équivalent connu

(25) Chat échaudé craint l'eau froide.

$$
\downarrow
$$

Un chat qui a été brûlé par de l'eau bouillante se méfie de l'eau, même froide

Le sens du proverbe (24) est donc interprété et la portée de ce dernier est effective $\downarrow$

« On craint jusqu'à l'apparence de ce qui fait souffrir » ou bien encore « Toute expérience malheureuse doit servir de leçon de prudence »

Afin de prouver le bien-fondé de cette démonstration, nous avons soumis à un nouveau panel d'informants (deux groupes de quinze personnes chacun) les deux tests suivants : 


\section{Test 1}

De quel proverbe français connu rapprocheriez-vous l'énoncé suivant :

«Celui qui s'est brulé avec la tchorba* souffle jusque dans le yaourt » (traduction d'un proverbe roumain)

Justifiez votre réponse en quelques mots.

*La tchorba est une soupe de boulettes de viande très relevée (recette bulgare)

\section{Test 2}

De quel proverbe français connu rapprocheriez-vous l'énoncé suivant :

«Celui qui s'est brulé avec la tchorba* souffle jusque dans le yaourt» (traduction d'un proverbe roumain)

$\square$ L'habit ne fait pas le moine

$\square$ Tant va la cruche à l'eau qu'à la fin elle se brise

$\square$ Chat échaudé craint l'eau froide

$\square$ Qui vole un œuf vole un bœuf

$\square$ Rien ne sert de courir il faut partir à point

*La tchorba est une soupe de boulettes de viande très relevée (recette bulgare)

\section{Résultats test 1}

\begin{tabular}{|c|l|c|c|}
\hline \multicolumn{2}{|c|}{$\begin{array}{c}\text { Proverbes connus proposés } \\
\text { fréquence et pourcentage }\end{array}$} & \multicolumn{2}{c|}{ Résultats } \\
\hline$(25)$ & Chat échaudé craint l'eau froide & 11 & $76 \%$ \\
\hline$(26)$ & Qui s'y frotte s'y pique & 1 & $6 \%$ \\
\hline$(27)$ & Qui se marie à la hâte, se repent à loisir & 1 & $6 \%$ \\
\hline$(28)$ & Il n'y a pas de fumée sans feu & 1 & $6 \%$ \\
\hline$(29)$ & Si tu bois un laxatif cul-sec, il ne le restera pas longtemps & 1 & $6 \%$ \\
\hline
\end{tabular}

\section{Résultats test 2}

\begin{tabular}{|c|l|c|c|}
\hline \multicolumn{2}{|c|}{$\begin{array}{c}\text { Proverbes connus proposés } \\
\text { fréquence et pourcentage }\end{array}$} & \multicolumn{2}{c|}{ Résultats } \\
\hline$(6)$ & L'habit ne fait pas le moine & 0 & $0 \%$ \\
\hline$(7)$ & Tant va la cruche à l'eau qu'à la fin elle se brise & 1 & $7 \%$ \\
\hline$(25)$ & Chat échaudé craint l'eau froide & 12 & $80 \%$ \\
\hline$(5)$ & Qui vole un œuf vole un bœuf & 0 & $0 \%$ \\
\hline$(5)$ & Rien ne sert de courir il faut partir à point & 2 & $13 \%$ \\
\hline & & $\mathbf{1 5}$ & $\mathbf{1 0 0} \%$ \\
\hline
\end{tabular}

Les résultats sont probants. Dans les deux cas, les informants orientent, pour la grande majorité, leur choix vers le proverbe attesté (25) Chat échaudé craint l'eau froide. 
Afin d'observer si le raisonnement opéré pour rapprocher les deux énoncés convoquait chez les locuteurs francophones les mêmes concepts, les mêmes analogies, donc mêmes savoirs encyclopédiques que ceux précédemment exposés, nous avons demandé aux personnes interrogées pour le test 1, de justifier leur proposition en quelques mots. Il faut souligner que les justifications pour l'énoncé (25) sont unanimes; voici les réponses données :

\begin{tabular}{|c|l|}
\hline \multicolumn{2}{|c|}{ Justifications } \\
\hline (25) & $\begin{array}{l}\text { Analogie : tchorba = chaud / yaourt = froid } \\
\text { Si on se brûle avec de la soupe on souffle même sur du yaourt comme le chat qui s'est } \\
\text { brûlé se méfie de l'eau froide. } \\
\text { On se méfie de tout après une mauvaise expérience. }\end{array}$ \\
\hline$(26)$ & Analogie : brûlure = frotter donc piquer / yaourt = la durée de la douleur \\
\hline$(27)$ & $\begin{array}{l}\text { Brûlure = Mariage hâtif = passion brûlante = aveuglement } \\
\text { yaourt = pas loisir, mais le repentir = la prise de distance, donc à la froideur du raison- } \\
\text { nement }\end{array}$ \\
\hline$(28)$ & $\begin{array}{l}\text { L'idée qu'une situation en implique une autre (cause, conséquence) tchorba = feu / } \\
\text { yaourt = fumée }\end{array}$ \\
\hline$(29)$ & Tchorba = plat épicé / conséquence = troubles digestifs \\
\hline
\end{tabular}

L'idée de cette compétence parémique qui se traduit en réception, entre autres sinon par compréhension d'un énoncé émanant d'une culture différente en interprétant toute la « charge sémantique » qu'il contient, à tout le moins de pouvoir reconnaître l'énoncé source comme étant un proverbe et de le rapprocher d'un proverbe attesté dans notre idiolecte. Ce processus interprétatif passe bien par la convocation d'un savoir encyclopédique :

- soit par analogie de concept ici : (25) tchorba/brûler = chaud = chat échaudé $/ /$ yaourt $=$ froid $=$ eau froide; et par glissement : méfiance de tout (yaourt) après une mauvaise expérience (brûlure avec la tchorba);

- soit par analogie des sensations évoquées si l'on prend le sens littéral des deux énoncés : (26) brûlure/piqure; et qui, transposé au sens profond, garde le même rapport analogique - évocation du danger, de la sensation désagréable;

- soit par analogie de valeur sémantique : (28) relation d'implication : Si P alors Q (cause/conséquence);

- soit par référence au savoir encyclopédique afférant que ce soit littéralement (référence au plat typique qu'est la tchorba (29)) ou de façon imagée (27).

Si plusieurs énoncés ont été proposés, les proverbes convoqués sont globalement orientés vers la même représentation sémantique, à fortiori sur la représentation des concepts « brûlure = chaud » / « yaourt = froid ». Ainsi les énoncés soumis aux informants comme (5) ou (6) n’ont pas été retenus comme équivalents possibles, ni des proverbes comme (9) ou (11) pourtant considérés par bon nombre comme étant prototypiques (cf. question I de 
l'enquête) de la notion qui auraient pu satisfaire en certains points comme tels (cf. implication, l'idée de danger). Pourtant, le locuteur fait automatiquement ou instinctivement la part des choses du point de vue du sens de l'implication supposée par les énoncés suivants :

(24) brûlure avec la tchorba $>$ yaourt

(25) chat échaudé > eau froide

\section{alors que :}

(5) voler un œuf $<$ voler un bœuf

(11) semer le vent $<$ récolter la tempête

La dimension d'intensité exprimée par l'implication est ici représentée par « <» lorsqu'elle est croissante et par «>» lorsqu'elle est décroissante.

Si l'on prend les exemples (6) L'habit ne fait pas le moine ou (9) Pierre qui roule n'amasse pas mousse, le locuteur n'aura pas à l'esprit de convoquer ces occurrences pour lui permettre d'accéder au sens profond de l'énoncé (24), puisque spontanément, il sait que les connaissances sémantiques inhérentes à ces énoncés ne lui permettront pas d'y parvenir :

(6) L'apparence peut être trompeuse ou Il faut s'abstenir de ne juger les gens qu'à leur apparence.

(9) L'instabilité est un obstacle à tout enrichissement ou au contraire une incitation à garder une certaine dynamique, un mouvement quasi perpétuel pour empêcher tout engourdissement (matériel ou intellectuel).

Après avoir compulsé tous les résultats de l'enquête, nous avons répertorié, dans Le dictionnaire des proverbes et dictons (1993), Le Robert et via Internet, différentes variantes des énoncés (24) et (25), afin d'élargir nos observations vers la mémoire culturelle.

\begin{tabular}{|c|c|c|}
\hline Pays d'origine & Proverbe d'origine & Traduction littérale en français \\
\hline Allemagne & Ein gebranntes Kind scheut das Feuer & Un enfant brûlé craint le feu \\
\hline Angleterre & A burned child dreads the fire & Un enfant brûlé redoute le feu \\
\hline $\begin{array}{l}\text { Angleterre / } \\
\text { États-Unis }\end{array}$ & Once bitten, twice shy & Une fois mordu, deux fois plus craintif \\
\hline Argentine & $\begin{array}{l}\text { El que se quema con leche, ve una vaca } \\
\text { y llora. }\end{array}$ & $\begin{array}{l}\text { Celui qui se brûle avec du lait, voit } \\
\text { une vache et pleure }\end{array}$ \\
\hline Brésil & Gato escaldado tem medo de água fria & Chat échaudé craint l'eau froide \\
\hline Bulgarie & Парен каша духа & $\begin{array}{l}\text { Une fois brûlé, on souffle sur la } \\
\text { soupe. }\end{array}$ \\
\hline Côte d'Ivoire & $\begin{array}{l}\text { Lorsqu'un serpent te mord et que tu } \\
\text { vois le ver de terre, tu te méfies de lui. }\end{array}$ & \\
\hline Danemark & Brændt barn skyr ilden & Enfant brûlé évite le feu \\
\hline Égypte & اللى اتلسع من الشوربة ينفخ فى الزبادى & $\begin{array}{l}\text { Étant brûlé par du potage, souffle } \\
\text { dans le yaourt }\end{array}$ \\
\hline
\end{tabular}




\begin{tabular}{|c|c|c|}
\hline Espagne & Gato escaldado, del agua fría huye & Chat échaudé fuit l'eau froide \\
\hline Espagne & $\begin{array}{l}\text { El que se quema con leche, cuando ve } \\
\text { una vaca llora. }\end{array}$ & $\begin{array}{l}\text { Celui qui s'échaude avec du lait, } \\
\text { quand il voit une vache, il pleure. }\end{array}$ \\
\hline Italie & $\begin{array}{l}\text { Can scottato dall'acqua calda teme la } \\
\text { fredda }\end{array}$ & $\begin{array}{l}\text { Chien brûlé par l'eau chaude craint } \\
\text { la froide }\end{array}$ \\
\hline Liban & Yalli kewi el halib, bi nafikh 3al laban. & $\begin{array}{l}\text { Celui qui est brûlé par le lait, souffle } \\
\text { sur le yaourt. }\end{array}$ \\
\hline Roumanie & Cine s-a fript cu ciorbă, suflă şi-n iaurt & $\begin{array}{l}\text { Celui qui s'est brûlé avec de la } \\
\text { tchorba (= soupe), souffle même dans } \\
\text { le yaourt. }\end{array}$ \\
\hline Roumanie & Cand te frigi, sufli si in iaurt! & $\begin{array}{l}\text { Une fois brûlé, on souffle même dans } \\
\text { le yaourt! }\end{array}$ \\
\hline Russie & $\begin{array}{l}\text { Обжегшись на молоке, дуют на воду } \\
\text { / будешь ду }\end{array}$ & $\begin{array}{l}\text { Après avoir brûlé sur le lait, on } \\
\text { souffle sur l'eau. }\end{array}$ \\
\hline Tunisie & Elli klé degga biha mét & Celui qui a été piqué en est mort \\
\hline Uruguay & $\begin{array}{l}\text { El que se quema con leche ve la vaca } \\
\text { y llora }\end{array}$ & $\begin{array}{l}\text { Celui qui s'est brûlé avec du lait voit } \\
\text { la vache et pleure }\end{array}$ \\
\hline
\end{tabular}

La « mémoire culturelle » quant à elle laisse une part de liberté au locuteur, en sont pour preuve les nombreuses variantes en production, comme en réception (interprétation des énoncés proverbiaux en langue maternelle ou culture commune).

Cette part de liberté se démontre principalement par le choix :

- de la syntaxe : (23) Il faut battre le fer tant qu'il est chaud / quand il est chaud;

- du lexique : (7) Tant va la cruche à l'eau qu'à la fin elle se cassel brise;

- de la ponctuation : (8) Rien de sert de courir (,) il faut partir à point.

- de la variante régionale : (25) À la Sainte-Luce, le jour crô̂t du saut d'une puce.

(26) À la Sainte-Luce, les lumières brillent. En référence à la traditionnelle fête des lumières de Lyon (savoir encyclopédique).

Ce fonctionnement de la compétence parémique expliqué ci-dessus, fait directement référence à Carl Jung et son inconscient collectif.

[...] Les instincts et les archétypes constituent l'ensemble de l'inconscient collectif ${ }^{5}$. Je l'appelle « collectif » parce que, au contraire de l'inconscient personnel, il n'est pas fait de contenus individuels plus ou moins uniques ne se reproduisant pas, mais de contenus qui sont universels et qui apparaissent régulièrement [...] sont des connexions mythologiques, des motifs et des images qui se renouvellent partout et sans cesse, sans qu'il y

5. L'archétype est une forme a priori de toute expérience humaine, inscrite dans la structure du cerveau et conditionnant tout schéma de pensée ou de représentation constituant l'ensemble de l'inconscient collectif. 
ait tradition ni migration historique. Ainsi, les influences de l'inconscient collectif, lorsqu'elles impriment le conscient, sont à la source des courants de croyance, des expériences religieuses, des visions extatiques mais aussi des arts, de la littérature et des rituel. En ce sens il apparaît comme inconstant pour le sujet alors que, dans sa nature profonde, il ne se transforme jamais. (Carl Jung, Métamorphoses de l'âme et ses symboles. Analyse des prodromes d'une schizophrénie, 1950.)

En somme, ce lien entre compétence parémique et mémoire sémantique / mémoire culturelle peut se résumer comme suit :

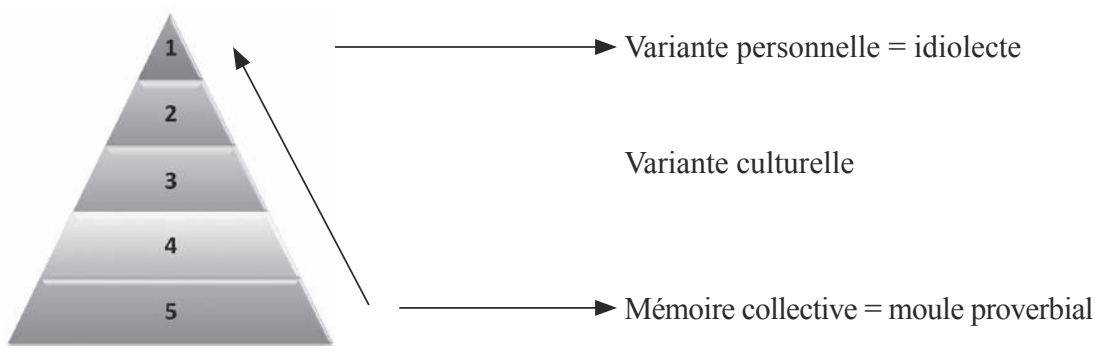

Représentation conique de la structure de la psyché selon la psychologie analytique :

1. le Moi;

2. le conscient;

3. l'inconscient personnel;

4. l'inconscient collectif;

5. la partie de l'inconscient collectif qui ne peut être connue, dite « inconscient archaïque ».

Ainsi la compétence parémique s'acquiert, se développe et se façonne indissociablement de l'expérience langagière. En réception elle convoquera essentiellement la mémoire collective, alors qu'en création elle s'appuiera principalement sur la mémoire individuelle.

\section{Conclusion}

Nous allons pour clore élargir et en même temps rassembler les raisonnements et les démonstrations qui précèdent.

Pour Lakoff et Turner (1989: 162), le proverbe est un exemple de métaphore ou d'utilisation de la métaphore : LE GÉNÉRIQUE, C'EST DU SPÉCIFIQUE. À savoir par là qu'on projette une situation ou un schéma spécifique de départ (situation source) (par ex. "L'aveugle maudit le fossé », Blind / Blames the ditch) sur toute une série de situations, innombrables et tout aussi spécifiques (situations cibles), si bien que le schéma source acquiert cette dimension générique. Ainsi, les proverbes nous renseignent et sur le monde et sur l'ordre des choses, qu'ils illustrent, et, ce qui est le propre des proverbes parmi les EP, sur la place de l'homme dans cet ordre, ou cette chaîne ou échelle; car en parlant de l'homme et de ses expériences, 
par exemple sous la figure d'une grenouille ou d'un couteau ${ }^{6}$, ce sont les maillons de la chaîne et les étages de l'échelle que nous mettons en contact (Lakoff et Turner : 166).

Loin d'être un énoncé figé et proposant une seule forme attestée immuable, le proverbe est un élément linguistique qui suit les évolutions de la langue. Il n'est pas appris par cœur, en sont pour preuve les variantes existantes d'une forme attestée, mais découle d'un apprentissage culturel, d'une connivence linguistique qui s'acquiert au même titre que le savoir sémantique.

Guy Achard-Bayle et Bertille Schneider
Université Paul Verlaine-Metz-CELTED

\section{Références}

Achard-Bayle, G., 2008, Les Réalités conceptuelles. Identité et/en fiction, Metz, Université de Metz, coll. « Recherches textuelles », $n^{\circ} 8$.

—, 2009, « Mémoire et reconnaissance : De Laudibus Provincice une chorographie du XVI ${ }^{\mathrm{e}}$ siècle. À la (re-)découverte d'un patrimoine », dans M.N. Fouligny et M. Roig-Miranda (dir.), Mémoire et découvertes : quels paradigmes?, actes du colloque international organisé à Nancy (15-17 novembre 2007), Nancy, PU Nancy, coll. « Europe XVI-XVII », n ${ }^{\circ} 13$ p. $157-175$.

Achard-Bayle, G. et Schneider, B., 2010, « Les énoncés parémiques, hypo- et paratactiques : des constructions syntaxiques aux interprétations sémantiques », dans M.-J. Béguelin, M. Avanzi et G. Corminbœuf (dir.), La Parataxe, t. II, Structures, marquages et exploitations discursives, actes du colloque de Neufchâtel (12-15 février 2007), Berne, Peter Lang, p. $95-120$.

Anscombre, J.-Cl., 2000, « Parole proverbiale et structures métriques », Langages, $\mathrm{n}^{\circ} 139$, p. 6-27.

—, 2003, « Les proverbes sont-ils des expressions figées? », Cahiers de lexicologie, $\mathrm{n}^{\circ} 82$, p. 159-174.

Arnaud, P. J. L., 1991, « Réflexions sur le proverbe », Cahiers de lexicologie, $\mathrm{n}^{\circ} 59$, p. 5-27.

6. Lakoff et Turner (184 et 207) : Frog / forgets he had a tail. Knife can't whittle / its one handle. 
Charolles, M., 1997, L'encadrement du discours univers, champs, domaines et espaces, Université Nancy 2.

Donald, M., 1991, Origins of the Modern Mind: Three Stages in the Evolution of Culture and Cognition, Cambridge, MA, Harvard University Press, http://cogweb.ucla.edu/Abstracts/Donald_91.html.

Eliade, M., [1957] 1965, Le Sacré et le Profane, Paris, Gallimard, rééd. coll. « Folio-Essais », 1987 et 2009.

Fauconnier, G., 1984, Espaces mentaux. Aspects de la construction du sens dans les langues naturelles, Paris, Minuit.

Gibbs, R. (ed.), 2008, The Cambridge Handbook of Metaphor and Thought, Cambridge, Cambridge University Press.

Gouvard J.-M., 1996, "Les formes proverbiales », Langue française, $\mathrm{n}^{\mathrm{o}} 110$, p. 48-63.

Gréa, P., 2002, « Intégration conceptuelle et métaphore filée, Langue Française, $\mathrm{n}^{\circ} 134$, p. 109-123.

Jolles, A., 1972, Formes simples, Paris, Seuil.

Kleiber, G., 1999, « Les proverbes : des dénominations d'un type “très très spécial" ", Langue française, $\mathrm{n}^{\circ} 123$, p. 52-69.

—, 2000, « Sur le sens des proverbes », Langages, n 139, p. 39-58.

—, 2006, « Histoire de couple : proverbes et métaphores », Communication au colloque Lexique-grammaire de Palerme (6-10 septembre).

Lakoff, G., 1987, Women, Fire, and Dangerous Things : What Categories Reveal About the Mind, Chicago, The University of Chicago Press.

Lakoff G. et Johnson M., 1980, Metaphors We Live By, Chicago, The Chicago University Press, trad. fr. François Récanati, 1985, Les métaphores dans la vie quotidienne, Paris, Minuit.

Lakoff, G. et Turner, M., 1989, More than Cool Reason. A Field Guide to Poetic Metaphor, Chicago, The Chicago University Press.

Le Goffic, P., 2002, « Marqueurs d'interrogation/indéfinition/subordination : essai de vue d'ensemble », Verbum, vol. 24, no 4, p. 315-340.

Lovejoy, A., 1936, The Great Chain of Being : A Study of the History of an Idea, Cambridge, MA, Harvard University Press, rééd. 2005, Harper \& Row.

Mayr, E., 1982, The Growth of Biological Thought. Diversity, Evolution and Inheritance, Cambridge, MA, Harvard University Press, trad. fr. 1989, Histoire de la biologie, rééd. 1995, Paris, Le Livre de Poche, coll. « Références ». Mejri, S., 2001, « La structuration sémantique des énoncés proverbiaux », L'information grammaticale, $\mathrm{n}^{\circ} 88$, p. 10-15.

Perrin, L., 2000, « Remarques sur la dimension générique et sur la dimension dénominative des proverbes », Langages, $\mathrm{n}^{\circ} 139$, p. 81-98. 
Schapira, C., 1999, Les Stéréotypes en français : proverbes et autres formules, Paris, Ophrys.

—, 2000, « Proverbe, proverbialisation et déproverbialisation », Langages, $\mathrm{n}^{\mathrm{o}} 139$, p. 81-97.

Suber, P., 1997, The Great Chain of Being, document de travail en ligne avec lien vers présentation Powerpoint, http://www.earlham.edu/ peters/ courses/re/chain.htm (consulté le 10 décembre 2009).

Tamba, I., 2000, « Formules et dire proverbial », Langages, n 139, Paris , Larousse, p. 110-119.

Turner, M., 1996, The Literary Mind. The Origins of Thought and Language, Oxford, Oxford University Press.

Victorri, B., 2006a, " À la recherche de la langue originelle », dans J.-L. Dessalles, Pascal Picq et Bernard Victorri (dir.), Les Origines du langage, Paris, Le Pommier.

—, 2006b, « Origine des langues et du langage », Version préliminaire pour l'Encyclopoedia Universalis, http://halshs.archives-ouvertes.fr/ docs/00/06/47/03/PDF/Origine_des_langues_et_du_langage.pdf (consulté le 10 décembre 2009).

\section{Références des exemples attestés}

Benoit, M. et Michel, C., 2001, Le Parler de Metz et du pays messin, Metz, Serpenoise.

Bérisé, C., 2001, Dictionnaire des expressions perdues, Paris, La Bruyère.

Delacourt, F., 1996, Proverbes, dictons et citations pour toutes les occasions de la vie, Paris, De Vecchi.

La Bonnardière, A.-M., 1975, Le Livre des proverbes, Paris, Études Augustiennes.

Le Dictionnaire des proverbes et dictons, 1993, Paris, Le Robert.

Le Dictionnaire des proverbes, 1980, Paris, Larousse.

Le petit Larousse illustré, 1990, Paris, Larousse.

La Fontaine, J., 1668, Fables.

Lanher, J. et Litaize, A., 1989, Au fil du temps : dictons de Lorraine, Nancy, Presses universitaires de Nancy.

—, 1990, Dictionnaire du français régional de Lorraine, Paris, Bonneton.

Mergnac, M.-O. (dir.), 2003, Mille proverbes et dictons de tous les temps, Paris, Archives et Culture.

Reitel, F. (dir.), 1981, La Tradition en Lorraine, Wettolscheim, Mars et Mercure, 5 vol. 


\section{Annexes (sources : Wikipedia)}

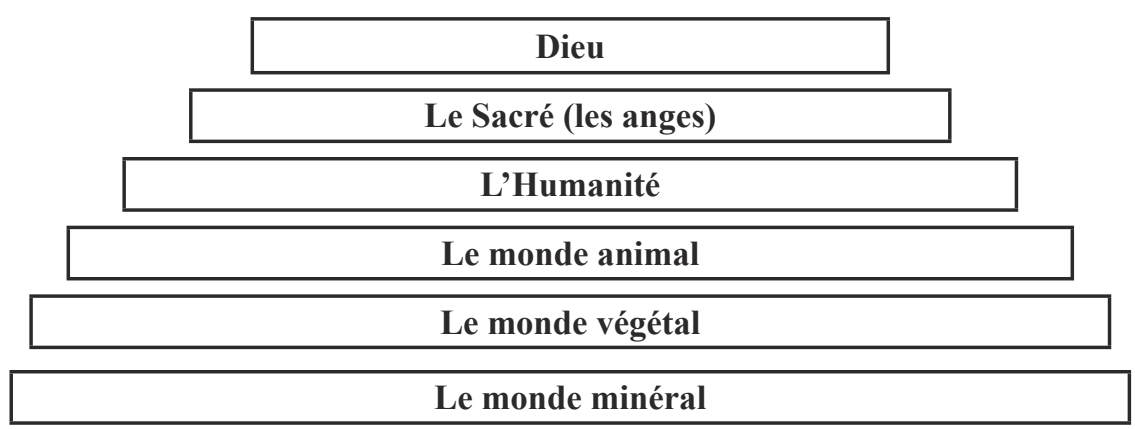

Classification centrale de la scala naturce

http://fr.wikipedia.org/wiki/Scala_natur\%C3\%A6

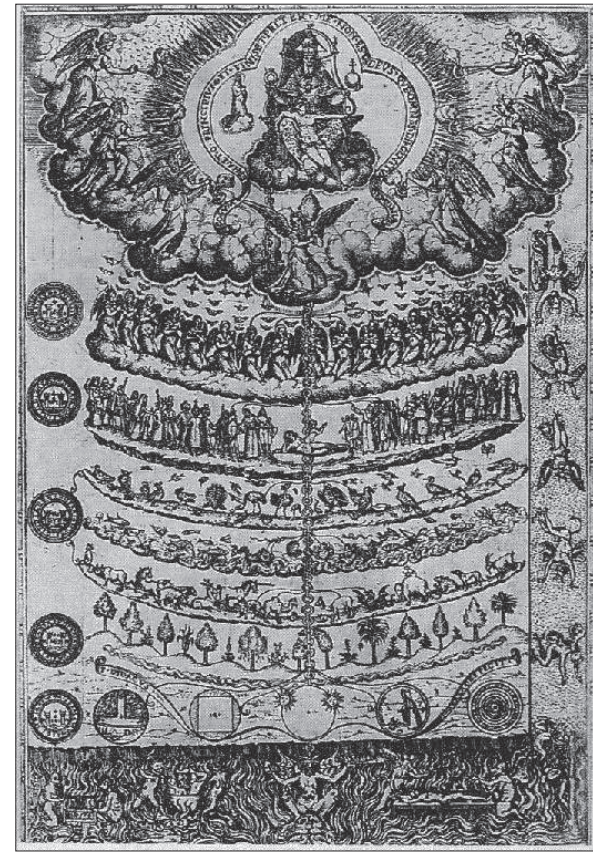

Grande Chaîne des Êtres

http://fr.wikipedia.org/wiki/Scala_natur\%CC3\%A6 


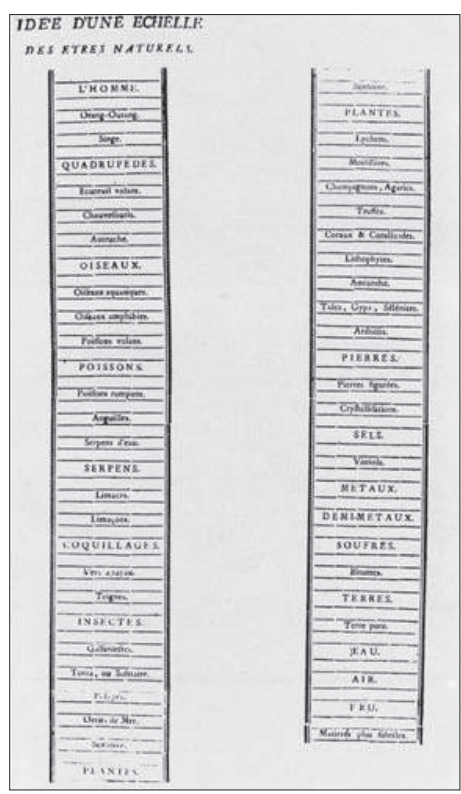

Charles Bonnet (1720-1793)

http://commons.wikimedia.org/wiki/File:Bonnet_echelle_des_etres_17.png 